\title{
Emotional-Dimension Based Musical Performance Study
}

\author{
1,a Wang-Manjun \\ ${ }^{1}$ Nanjing University of Science and Technology,Jiangsu,Nanjing,China,210008 \\ a402000897@qq.com
}

Keywords: Musical Performance, Artistry, Emotional Dimension, Positioning.

\begin{abstract}
In order to manage the conflict between the non-conceptual musical emotion and the conceptual music performance teaching, this paper discusses various performance dimensions separately like speed, strength, timbre etc. and two distinctive multidimensional models: performance worm model and Braunschweig Lens model, to research the common law between performance parameters and emotion performance. We conclude that the essence of musical expression lie in that various performance parameters show systematically joint deviation and regression.
\end{abstract}

\section{Introduction}

Most music performance learners and teachers tend to regard emotional expression ability in musical performance as a gift. In fact, we can treat this kind of issued in the scientific view. First of all, assuming that a song can be played in two ways, one is the rigid play completely according to the score, while another is various with rich expressive plays. To research the emotional factors in the latter, we can compare the acoustic characteristic differences between the two ways, and find the general characters of several individual in the latter. Secondly, from the view of musical performance, the acoustic characteristic differences in different music performance is nothing more than the differences in speed, strength etc. a series of parameters the performer adopts. If we can find these parameters changing rules based on the intrinsic callings of the scores (music) from those expressive performances, the emotional problems in music performance will become clear. Thus, it is necessary to conduct relatively independent discussion about the inner link between the main dimensions about expressive performances and the scores.

\section{Multi-Dimension Analysis in Musical Performance Acoustics}

Timbre.Timbre is another important parameter in performance. It is a constant as well as a variable. On the one hand, it is restricted by the attribute of the instrument and personal style of the performer, which is the relatively fixed in performance; on the other hand, it changes according to the intrinsic requirement in the process of playing. From the prospect of acoustics, taking the abundant and distinctive string music for example, there are two main dimensions about timbre change: vibration and high-frequency overtone. The former is related to the performance of vibrato while the latter is associated with Su(1Pontl'ceoll) and Su(1atsto). The vibrato affects the amplitude and frequency of the vibration, while Su(1Pontl'ceoll) and Su(1atsto) respectively make the timbre bright, bold or dark, hazy, namely increasing or decreasing the high frequency overtone from the view of acoustics. The changes of the dimension do not exist alone, which is usually with synchronized systematic offset Figure 1 shows the fragment of Repin G Minor 1st violin concerto, and analyzes the timbre change information by spectrogram when Menuhin played the cadenza in the beginning of Bruch G Minor1st violin concerto. Affected by the trend of the melody, harmony and music structure, timbre change is as well rich except for dimension changes like the rhythm and strength we have discussed. $\mathrm{t} 1$ shows the process of a prolonged sound fading away, and arch timbre change at $\mathrm{t} 2$ is similar in the push processing. The timbre changes of the point emphasize on the tensity of leading tone, and the dark tone opposite to the point is mainly done to keep the excitement behind. Point is the brightest tone, and the highest amplitude and frequency of the vibrato, as well as with the most emotional stress, which is gradually stabilizing with the relaxation of the rhythm. 


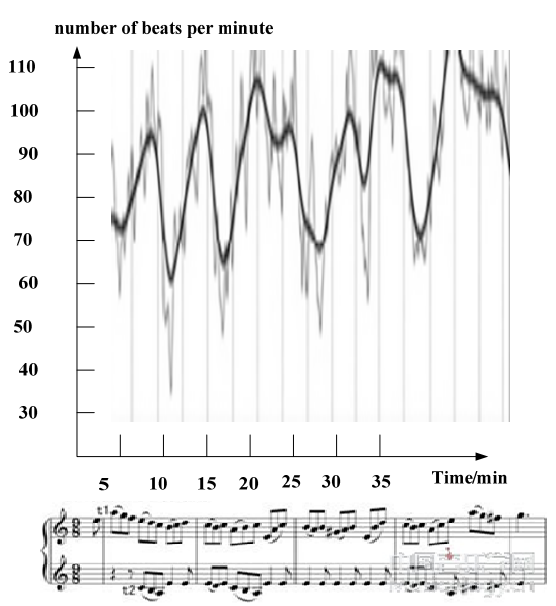

Figure1: Fragment of Bruch G Minor 1st Violin Concerto-Comprehensive Analysis of the Timbre Changes Spectrum

Volume and Strength. Except for the change of rhythm, performers have many other ways to show the music structure and the growth and regression of strength is one of them. When performing the first period of Mozart's sonata K.331, the two pianists divide the phase, knob, remit of the song very clearly by rhythm changes, and the changes of volume and strength mainly follow the similar rules. Two obvious fluctuates emerge at half cadence and the termination of the period. If we look closely, we will find smaller structures division units and corresponding fluctuation traces in the intensity curve. Figure 2 makes an obvious sense that this phenomenon of volume strength and micro-rhythm fluctuating with the music structure is similar with the mood and speed in sentence and phrase fluctuating, changing and phasing in oral reading. This will have significant applications in the 2.2.

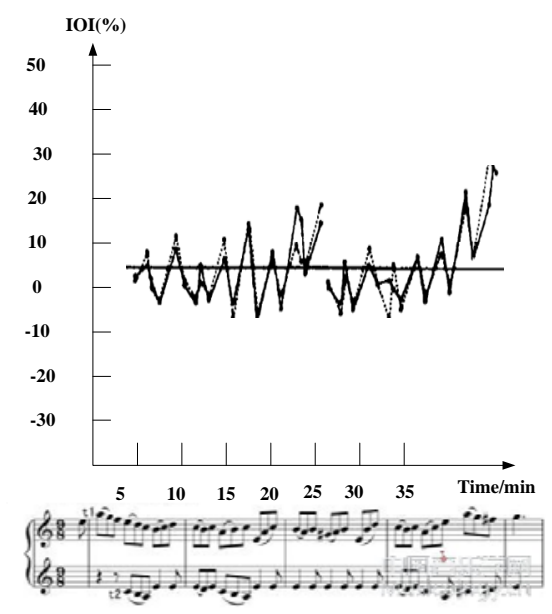

Figure2: Mozart: Piano Sonata K.331, Comprehensive Analysis of IOI Deviation and Strength

Speed and Rhythm. The duration of note is often played with different degrees of exaggeration; long note is dragged longer and short notes are played much shorter. As shown in Figure 3, when the two pianists playing this musical fragments, the performance duration of two sets of semiquaver in dash area shows obvious austerity comparing to other crotchets, and the austerity trend in the second group is much more distinct. Certainly, the playing speed of David Oistrakh is slower than Kogan's, and duration contrast is more exaggerate. When combining the mood of the music, he put more emphasis on the inner charm of the detailed structure of music: loose-intense-loose, loose-more intense- loose. 

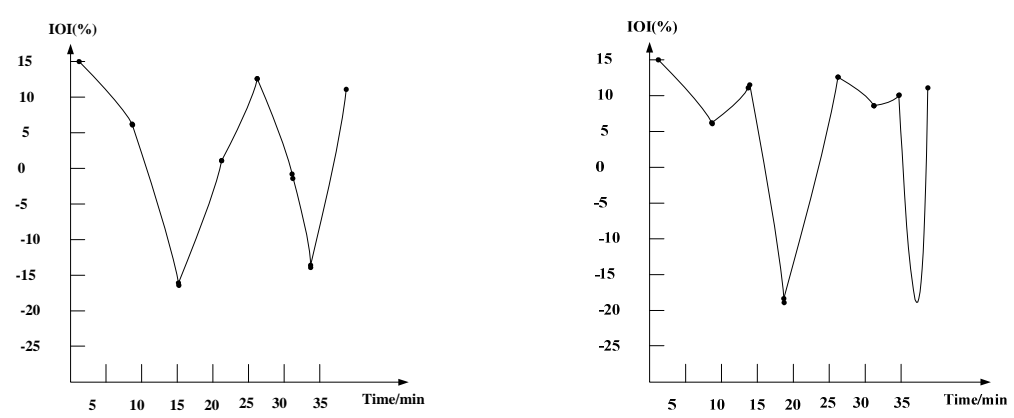

Figure3: Tchaikovsky: Violin Concerto, OP.35, IOI Analysis

\section{Positioning in Emotional Space by Multi-dimensional Performance Parameters}

Rhythm-Strength — time 3D Visualization Model. The rhythm and strength of this model can be put in a visualization model, which is wildly used in the pattern recognition research of musical expression and performance style. As shown in Figure 3, the performances of the two violin masters embody these features and have a lot of similarities. General trend of strength grows first before fading, and the speed moves forwards slightly before relaxing. The changes in speed and strength from bar 1 to3 is relatively temper, and bar 5 is the climax of the phrase, which is coincident with the intrinsic property of constrain-stretch—regression of this piece of music. The differences between the two people are that Parman makes more preparation in strength and rhythm for the following fluctuations, while Heifetz increases strength in the latter part of bar 4 and keep the gradually increasing trend to the first two pats of bar 5. The two people process differently in the climax near bar 5, Parman emphasizes on the lowest note of last shot of bar 4 and regards bar 5 as a whole to sing out coherently. Thus, there are almost no big strength changes except rhythm relaxing. Heifetz conducts motivated processing in the first 3 pats of bar 5 and hesitates at a tone of bar 4, from which the strength begins to drop. Afterwards, he made a rapid regression retain, preparing for the bigger joint changes of rhythm—strength in the latter half of bar 6. Parman's treating the phase end mainly reflects in strength.

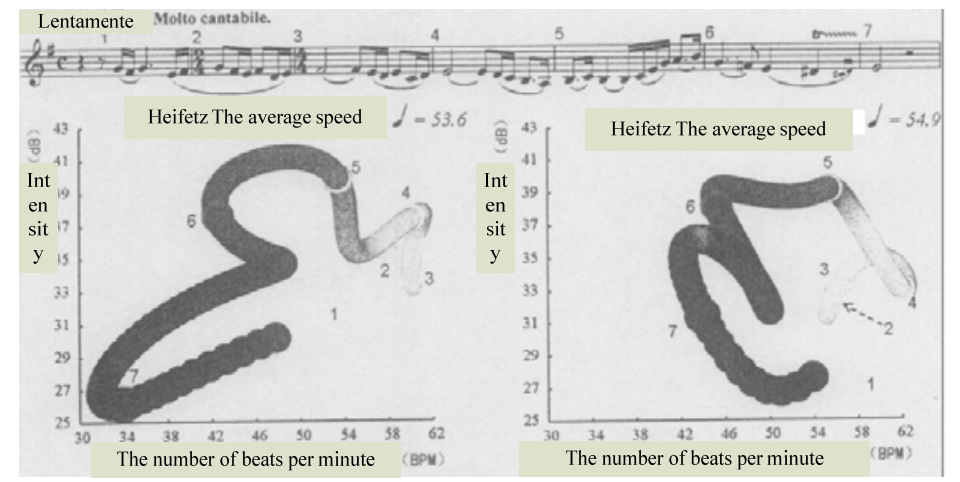

Figure4: Performance Worm Analysis

From the prospect of emotional space, worm model links the abstract space of performance parameters and virtual space of emotional movement, and represents the motion morphology of generalized emotion without good use of those conceptualized emotion nouns in life. The usual performance teaching is still dominated by oral delivering.

Braunschweig Lens Model in Musical Performance. Emotional expression in musical performance and language share close intercommunity and inheritance. However, the acoustics coding of language emotion does not require specialized acquired learning, while musical performance has complex techniques. Art emotions and life emotion are fundamentally unified and homologous. The latest research shows that the auditory coding human used in language expressive emotion is similar with the coding in musical performance. For instance, the characteristics of language expressing sorrow are slow tempo, weaker voice and relatively less energy of high frequency section in the voice frequency spectrum; and similar acoustic features are also used to express sorrow in musical performance. 
Lens model in musical performances is modified from the Brunswick lens model. As shown in Figure 4, the degree of accuracy refers to the proportional relation of performer's wills and audience's judges. Matching degree refers to the level of similarity of the prompt messages of performing and judging. Performers can express some kind of emotion to the audience accurately by acoustics, and performers and audience express and judge this emotion by the same performance prompt message. The information in Figure 4 can be understood in this way: performers and audience express and comprehend the angry by a series of common features coding like fast rhythm, grand volume, strong timbre, the pronunciation of staccato etc. Thus, the model is with high credibility.

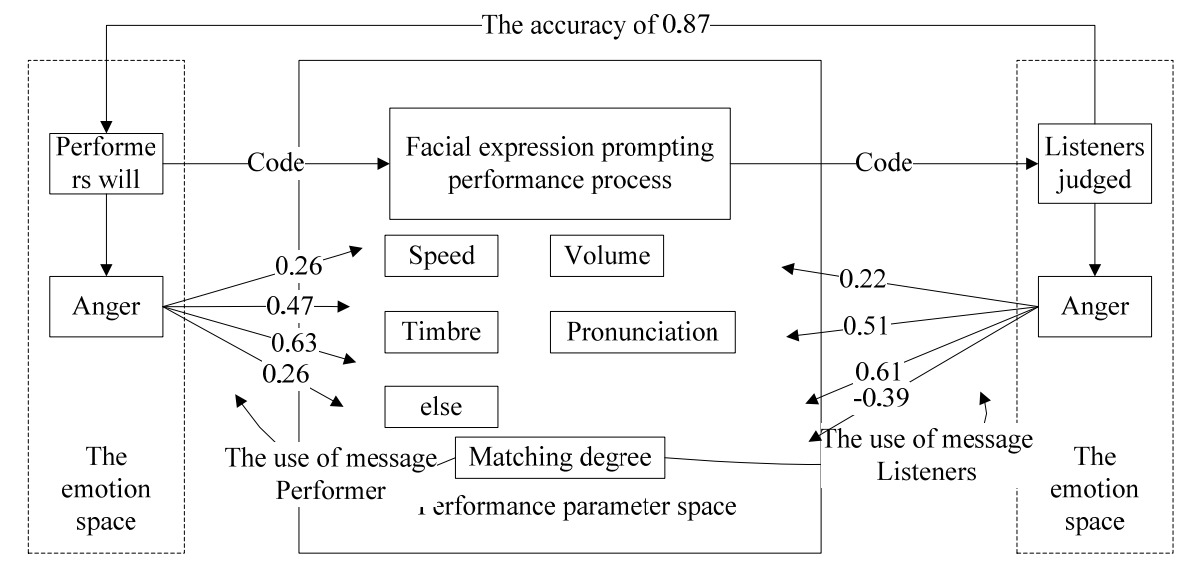

Figure 5: Braunschweig Lens Model of Emotional Expression in Musical Performance

The limitation of this model is also obvious. It has a distinct aesthetics tendency of reference. In most music, the contained emotions cannot be so clear to be concluded by one or two adjectives that the scope of practical application is relatively narrow. However, the thought of focusing and linking the emotional space of audience and performers by performing parameter lens is with enlightenment.

\section{Conclusion}

The emotional content of music does have the characteristics of unspeakable in its nature. However, since the didactical activities mainly depend on oral imparting, we have to objectify the subjective emotional experience to solve this pair of contradiction: the essence of emotional expression in musical performance lies in that various performance parameters show systematically joint deviation and regression; and scientific musical performance teaching process should contain the cognitive feedback reflecting the emotional connotation objectively and aiming at each performance parameters directly.

\section{Reference}

[1] Wang Site. Mental Connotation and Aesthetic Orientation in "Well-Tempered Clavier" of Bach[J]. Journal of Nanjing University of the Arts, 2003, 2:32-36.

[2] Huang Jianyun. Specialty and Beauty[M]. People's Publishing House, 2009.

[3] Sun Jiabin. Significance of Psychological Perception in Music Aesthetic[J]. Chinese Music, 2006, (2):114-116.

[4] Lin Fengqing. Position of Inner Musical Hearing and Its establishment in Musical Performance[J]. Journal of Guangxi Art Institute, 2005, (6):11-19

[5] Feng Xiaogang. Analysis on the Comprehension in Musical Performance[J]. Journal of Nanjing Arts Institute, 2008,(4):88-94.

[6] Li Chaohua. [J]. The Teaching of Emotion, Senior Emotion and Musical Performance Arts. Journal of Northwest University for Nationalities,2009,(12): 20-25. 
[7] Feng Xiaogang. Emotion and Experience in Music Comprehension Process [J1. Symphony, 2000, 3:40-45.

[8] Yang Jian. The Science of Music Expression and Understanding [J].Musical Instrument: 2004, 11:20-26.

[9] Wang Naige. Lens Model in Musical Emotional Communication[J].Advances in Psychology, 2003, 11(5):505-510. 